\title{
A New Model for a New Central Purchasing Unit for Health Products to Support Reaching the Objectives of a Lasting Development and Facing Sanitary Crisis
}

\author{
Alassane $\mathrm{Ba}^{1}$ and Karl HOUNMENOU ${ }^{2}$ \\ 1. PharmD, MHE, Director of the CHMP, Humanitarian Center of Pharmaceutical Practices, Clermont-Ferrand 63000, France \\ 2. Pharm D, MPH, Head of Pharmaceutical Expertise and Training in CHMP, Humanitarian Center of Pharmaceutical Practices, \\ Clermont-Ferrand 63000, France
}

\begin{abstract}
Medicines are a global, strategic and diplomatic issue [1]. The UN General Secretary's High Level Task Force [2] on Access to Medicines calls for a new approach to reduce the gap between medical innovations and access to medicines. Inequality in access to quality pharmaceutical products in a framework of global health democracy poses a threat to the ethical and equitable management of the provision of quality health care, particularly during global health crises. In French-speaking Africa, the lack of a unified production of pharmaceutical medicines forces wholesalers (public and private) to import almost all of their medicine demands resulting in a risk of dependence and major pharmaceutical quality defects. These central purchasing units are therefore often faced with the major challenge of guaranteeing the performance of their services and the security of their supplies. In order to achieve component 3 (“Access to Health") of the SDOs (sustainable development goals), in particular the access to affordable medicines, it is essential for a country to have a strategy of pharmaceutical independence [3] by anticipating the epidemiological transition and the management of health crises. This strategy of pharmaceutical independence is based in the short and medium term on the strengthening of central purchasing units and the establishment of a public-private partnership between central purchasing units and wholesalers, and in the long term on the establishment of competitive production units that comply with international standards. However, recent health crises have shown the fragility of public central purchasing units and make it difficult to anticipate health crises.
\end{abstract}

Key words: Medicines, central purchasing unit, access, pharmaceutical products.

\section{Introduction}

The legal model of central purchasing units in the French-speaking Black-Africa has always been the subject of many debates. But it must be assumed that there is no unique legal model that can be adapted to all countries and contexts. We must also accept that a model that was appropriate for an epidemiological context dominated by infectious diseases, where there was no access to information and communication technologies, is no longer appropriate for the current context of a health crisis, of transversal financing and of the obligation of accountability to patients. A review on the legal forms for central purchasing units carried

Corresponding author: Alassane Ba, Ph.D, Master, research filed: health economy. out by ACAME (Association of Central purchasing unit of essential medicines) in 2015 made it possible to map all possible legal forms and the feasibility of their application.

The objective of this document is not discussing legal forms, but to describe the fundamental organizational elements that should enable central purchasing units to play their full role as public health actors, in a context of epidemiological change and scarcity of financial resources.

Whatever the legal form and the organizational model, the central purchasing unit will not be able to avoid mastering a certain number of vital or even risky functions for its organization. An efficient and secure central purchasing unit a proactive centre, i.e. one capable of anticipating patient needs and monitoring 
information in order to anticipate crises, with an appropriate and practical organization and legal form that is focused on patients needs. [4]

It is also an almost industrial organization whose process and quality must be controlled and reliable over time. Working in the field of access to quality medicines, the central must act to guarantee the quality of medicines in order to protect the patient. [5]

\section{Materials and Methods}

To do so, it must ask itself the following questions:

1. What is the degree of control of the needs of health structures and what degree for an epidemiological evolution?

2. What are the risks of a health crisis?

3. What is the level of control for the processes of supply and logistics?

4. What is the organizational level to be able to face health crises?

In addition, the unit is evolving in a context of:

1. Major fluctuations in the pharmaceutical environment (globalization of trade);

2. Change in procurement rules at both national and international levels;

3. Increasingly binding (but justified) technical recommendations by the international organizations and development partners;

4. Globalization of the scourge of falsified medicines;

5. Regional and global health risks, etc.

The central purchasing unit must, therefore, identify the different pressures that the environment is exerting over it; this will allow the organization to adapt and orient its model.

\section{Results}

Serge Barbereau, in his article "Provisioning of Medicines in Developing Countries: We Must Save the Public Central Purchasing Units of Medicines in Africa” [6], had already alarmed about the subject of the central purchasing units.
He had shown that the low performance of central purchasing units depends on many external parameters that disturb the operation.

The performance of the central purchasing units can only be measured in terms of the quality of their logistics operations and good execution. Beyond the forecasting and planning processes (procurement, supplier control) which allow resources and means to be dimensioned, the capacity to deliver quality products at the operational level is essential, because it is only at this level that the efforts made can be materialized.

The central purchasing unit implements and uses many resources. Therefore, anticipating their use and management is crucial to meet patients needs and this through a logic of economic optimization, compliance with therapeutic protocols and the requirements of various donors and technical partners.

Central purchasing units management should focus on tactical resource planning and the means that allow the availability of an annual procurement plan with known delivery times negotiated with each manufacturer.

The operational production (controls the different stages of the pharmaceutical supply and logistics chain) of the service is the final step in a chain of upstream decisions and actions that can be jeopardized if operational excellence is not achieved. The delivery of an order on time and under the agreed conditions is the final visible operation.

\section{Discussion}

"When an unusual emerging event goes beyond the scope of day-to-day management of the alerts, given its scale, gravity or media nature, and may require coordination with many highly mobilized actors, the health authority sets up a specific and proportionate organization to ensure the follow-up and management of the event in question.”

The central purchasing unit is, therefore, part of the coordinated response to this crisis by providing an 
anticipation strategy for:

- The purchase, storage and distribution of pharmaceutical products that are not manufactured at the national level, in order to avoid stock shortages.

- The process, on behalf of the State, of the purchase, the management, the distribution and the elimination of the regional strategic stockpile of medicines and health products, which must be distributed rapidly.

For the implementation of this strategy, the central purchasing unit's strategy includes two essential dimensions:

- Coordination and management of pharmaceutical logistics operations (reinforcement of available human resources to cope with the event and avoid saturation of the existing system).

- Rapid negotiation with the authorities and financial partners on the emergency funding mechanisms (to ensure the immediate availability of necessary logistical tools, personnel and health products).

At the practical level, the central purchasing unit should:

1. Make themselves useful by taking the lead in procurement to help the populations effectively;

2. Simplifying its supply and distribution system so that the management cycle becomes the most efficient possible;

3. Make itself flexible to adapt to crisis management strategies; improve the quality of its management and logistics data to be able to deliver anticipated informed decision making;

4. Provide an acceptable platform for all stakeholders (national and international partners);

5. Improve its representativeness in a crisis area level; be prompt to intervene in time in crisis areas;

6. Guarantee administrative and financial stability during the crisis period.

\section{Conclusions}

Medications are a public health issue, an industrial, commercial and social product but also a resource for international political competition.

The advent of generics and rapid dissemination should enable the poorest populations to have access to priority and essential medicines. However, the limited resources of developing countries have exacerbated societal imbalances (middle class, poor, urban and rural populations, etc.) and have also led to questions about the capacities of health systems to ensure comprehensive and integrated care for all strata of society.

The policy of a worldwide and global (OMC, ADPIC) increases the sources of supply, of distribution and complicates the circulation of medical products by transcending the borders of countries.

In Africa, the creation of community spaces (UEMOA, CEMAC) guaranteeing the free movement of people and goods certainly facilitates access to medicines; however, it favors the trafficking of medical products at permeable borders and unfortunately allows the development of the illicit market and the counterfeiting of medicines in the continent.

The recent health crises (Ebola, Chikungunya, Zika), the epidemiological transition, the fight against cross-border epidemics (dengue, poliomyelitis, measles), require the authorities of developing countries to reflect on the accessibility and availability of quality medicines in a logic that integrates existing inequalities between different social classes.

One of the cornerstones of this availability and supply of quality and accessible medicines, therefore, remains in local production, alongside with the strengthening of the skills and prerogatives of public central purchasing units.

\section{References}

[1] The quality of medicines for the developing countries AMIPS N 68 -2004.

[2] 2016. Promoting Innovation and Access to Health Technologies. Report of the United Nations Secretary-General's High-Level Panel on Access to Medicines. 
A New Model for a New Central Purchasing Unit for Health Products to Support Reaching the Objectives of a Lasting Development and Facing Sanitary Crisis

[3] Succeeding calls for public health project, Tropical Medicine 6th edition, 2012.

[4] The Customer-Supplier Quality Charter STP-PHARMA PRACTICES, Volume 13, No. 13, May / June 2003.

[5] Good Pharmaceutical Distribution Practices STP
PHARMA PRACTICES, Volume 14, No. 1, January / February 2004.

[6] Barbereau, S. 2013. "Medicine Supplies in Developing Countries: Saving Public Medicine Procurement Bodies in Africa.” Médecine et Santé Tropicales 23: 251-5. 